\title{
Spatial memory exclusivity: Examining performance of multiple object-location memories
}

\author{
Thomas J. Dunn \\ Bishop Grosseteste University \\ Thom Baguley \\ Nottingham Trent University \\ Andrew K. Dunn \\ Nottingham Trent University
}

Research on location memory suggests that integration of separate sources of information does not occur when recalling the position of a common target object. In a relatively simple task, previous research shows no observable benefit from holding two spatial memories compared to one. It has been suggested that exclusively utilising only one of two memories may account for this finding. The current research tests the robustness of this idea as well as an alternative in the form of an averaging approach to combining spatial information. The results suggest that exclusivity may not be the best account for multiple spatial memory performance. Rather, memories may well combine in a manner similar to averaging, where information is available for each memory but combined in a non-beneficial way.

\section{Introduction}

A number of findings suggest the intuitive ease with which we integrate information to form spatial representations (e.g., a route back home, locating a parked car) may obfuscate a fragility that lies behind achieving such feats (Alvarez \& Cavanagh, 2004; Baguley, Lansdale, Lines, \& Parkin, 2006; Clark, Dunn, \& Baguley, 2013). Research on location memory suggests that integration of separate sources of information does not occur when recalling the position of a common target object (Baguley et al., 2006; Clark et al., 2013). This raises an interesting question as to the underlying cognitive processes and effectiveness of combining spatial information from memory.

Baguley et al. (2006) and Clark et al. (2013) sought to explore whether two independent sources of spatial information for the location of a common target can in some way support more precise recall of that target. Under the Baguley et al. (2006) paradigm, participants would encode the location of a target object in relation to either a single reference point (termed an 'anchor point') ${ }^{1}$ or in relation to two reference points on separate occasions. This led to individuals holding either a single representation or two separate representations for a target's location. Results from several experiments suggest that in this relatively simple task there is no observable benefit from holding multiple spatial memories (Baguley et al., 2006; Clark et al., 2013).
Baguley et al. (2006) put forward a parsimonious explanation of their findings by way of an exclusivity hypothesis. They suggest that given the opportunity to encode two spatial memories, neither integration nor selection of a more precise memory occur, instead only one memory is exclusively relied upon (i.e., encoded or retrieved). This finding is particularly interesting in light of contrasting reports of integration and non-integration in the literature (Rubin \& Wallace, 1989; Jones, 1987), especially within the field of spatial cognition (Molet, Bugallo, \& Gambet, 2011; Molet, Gambet, Bugallo, \& Miller, 2012; Alvarez \& Cavanagh, 2004; Baguley et al., 2006; Clark et al., 2013). Possible accounts for why two spatial memories do not surpass performance of a single memory are discussed.

\section{Exclusivity}

Exclusive processing is one strategy that can account for the equal performance observed when having the opportunity to utilise one or two-memories. If only one of two memories is randomly relied upon to make a spatial judgement, then the accuracy of such a judgement should approximate that of a single memory. This explanation suggests that during encoding or retrieval of two related spatial memories, one of the memories is either intentionally untapped or somehow blocked (e.g., Morris, 1981). In either case, this would result in an unsystematic and disproportionate degradation of spatial information contained within one of the two memories. In order to explicitly test this idea, the availability and 'quality' of information contained within both memories would need to be examined. However, under the Baguley et al. (2006) paradigm overall location memory was tested via concurrent cueing of the target object (i.e., both anchor points were presented together). This meant only the sum of location information derived from both sources was assessed. A simple adaptation of this design, namely by cueing both

\footnotetext{
${ }^{1} \mathrm{An}$ anchor point is defined as a point in figural space from which the spatial judgement of a target object can be made. For example, if a task involved judging the location of a target object such as a kettle in relation to a toaster, the toaster would be classed as an anchor point from which the spatial location of the target (i.e., kettle) could be judged.
} 
memories on separate occasions, would allow for explicit examination of memory degradation and thus scrutinise further the idea of exclusive processing.

Using a comparative spatial judgement task, Lansdale, Humphries, and Flynn (2013) provide some evidence that exclusive encoding may not be the best explanation of previous findings. In the Lansdale et al. (2013) task, individuals were required to store two separately learned spatial quantities (e.g., distance from an anchor point to target object), for the purpose of comparison at recall. The fact that the task was possible and subjects' performance was better than chance, indicated that two spatial representations were being held simultaneously. This suggests that both memories in the Baguley et al. (2006) task were also more than likely encoded at learning. However, this does not immediately preclude the idea that exclusivity could be occurring at retrieval. In fact, Lansdale et al. (2013) argue that exclusivity could well be cognitively efficient because it involves fewer operations and delivers greater accuracy when recall is laden with imprecision. They suggest that memory may function on a continuum from exclusivity through to additivity ${ }^{2}$ and accuracy is dependent upon the overall availability of traces and the degree of precision of location memory. They theorize that when precision is high, the use of two memories should improve overall accuracy (i.e., additvity). In comparison, when imprecision is introduced exclusivity might be favoured as a more efficient use of memory. Either way, Lansdale et al. (2013) provide a basis that supposes exclusive processing might play a role in tasks involving multiple sources of spatial information. However, what is not directly considered in previous work is the idea that information is utilised from both memories to inform spatial judgement, but does not equate to any observable improvement in performance. In other words, imprecision may not prevent integration occurring per se but rather negate any potential advantage from utilising two spatial memories.

\section{Averaging across memories}

In general, previous literature has tended to assume that integration equates to an increase in performance (Greenauer, Mello, Kelly, \& Avraamides, 2013; Molet et al., 2011; Rubin \& Wallace, 1989; Hollingworth \& Henderson, 2002; Jones, 1987; Bryant \& Subbiah, 1994; Lindberg \& Garling, 1987; Baguley et al., 2006; Clark et al., 2013). In light of this, the possibility that information is combined from two memories but does not result in any gains, has tended to be overlooked (Baguley et al., 2006; Clark et al., 2013). This next section discusses how combining information may vary in terms of its observable benefit.

Integration is thought to be productive when useful information from each memory is extracted and either independently or collectively utilised to point towards the same signal. A good example of this comes from Rubin and Wallace
(1989) who showed that two related routes (i.e., cues) to retrieving stored non-spatial information are greater than the arithmetic sum of each cue separately. Rubin and Wallace (1989) prompted subjects for a learnt word ('RED') with 'and/or' cues such as 'It's a colour' and/or 'It rhymes with bed'. They found recall was much better for the 'and' condition over the 'or' condition. In other words, multiple cues for the same memory were more efficient in extracting memory for the target word over and above the sum of its individual memories. Relating to the exclusivity effect, this suggests two processes must occur to replicate the multiple cue advantage as reported by Rubin and Wallace (1989). First, both memories should point to the same source (i.e., be acknowledged as being related) and second, a substantial amount of useful information must be collectively extracted and combined from both memories.

These possibilities sit neatly within a Bayesian account of spatial information integration (Cheng, Shettleworth, Huttenlocher, \& Rieser, 2007). In line with the idea of signal congruence, Cheng et al. (2007) argue that only when two sources of information stem from the same signal is it optimal to integrate them (weighting them in inverse proportion to their error). Alternatively, when both sources stem from different signals it is suboptimal to integrate them. Cheng et al. (2007) also suggest that one variety of combination involves the averaging of current and prior sources of information. They suggest that if uncertainty is apparent in a current source of information, then an optimal guess is the average from accumulated past experiences. Support for this idea comes from Hubbard and Ruppel (2000) who demonstrate spatial memory averaging occurs with stationary objects.

In the current study, the common target object - as the only universal feature from which both sources (memory representations) are derived - may be considered as the signal towards which both memories must point in order to be commensurate with beneficial combining. As a consequence of the Baguley et al. (2006) design, presenting the same target with different anchor points will inevitably result in each representation varying in terms of both spatial quantity (distance from anchor point) and vector information (direction from anchor to target). Combined, these aspects of the task design may potentially lead to sources of signal discrepancy and to uncertainty at recall when related cues are presented concurrently. Thus, integration (of a beneficial nature) may not occur at encoding due to signal discrepancy or at recall due to uncertainty (i.e., introduced via simultaneous cueing). In such circumstances an optimal strategy would be to average across accumulated past experiences and utilise information from both sources non-exclusively.

Previous theoretical standpoints have proposed that exclu-

\footnotetext{
${ }^{2}$ In this context, additivity implies that some form of integration has occurred forming a more precise representation greater than each memory in isolation.
} 
sivity (reliance on only one source of information) may be an efficient strategy that represents one end of a spectrum of processing determined by imprecision (Baguley et al., 2006; Clark et al., 2013; Lansdale et al., 2013). At the other end of the spectrum is additivity (Lansdale et al., 2013), where useful information from each memory is combined in an advantageous way. This approach suggests a switching must occur at some point (dependent on levels of imprecision) between relying on randomly sampling a single memory (exclusivity) to combining useful information from both memories (additivity). In contrast, an averaging approach posits that the same underlying strategy may occur across a spectrum of imprecision (dependent on levels of source uncertainty), only that as memories become more precise less error is also averaged (i.e., combined) and integration becomes tenable (i.e., additive). Thus, as imprecision declines, integration moves from being non-beneficial to an advantage, without the need to switch strategies.

In essence, non-beneficial averaging can be thought of as combining information by either equally weighting (and then averaging) precision and error from each memory representation or more generally averaging some component of memory that does not pertain to any accuracy advantage (e.g., spatial quantity). Either way, this would likely result in a memory representation that approximates performance of a single memory, across multiple observations.

\section{Current aims}

The current study sought to:

1) test the robustness of the idea that two competing spatial memories act exclusively by adapting the original Baguley et al. (2006) paradigm and examining information contained within each memory. It is hypothesized that if spatial information is present within both memories then exclusive retrieval may not be an appropriate explanation of how memories interact within the current task.

2) examine the plausibility that averaging across memories may explain previously observed one and two-memory performance. It is hypothesized that if the average accuracy of two separate memories approximates that of a singular memory that some form of non-beneficial combining may be taking place.

\section{Methods}

\section{Experimental paradigm}

Broadly, we followed the experimental design used by Baguley et al. (2006) and Clark et al. (2013) where exclusivity was initially observed. However, in order to test the idea that when given the opportunity to encode or retrieve two related spatial memories only one is relied upon (i.e., the exclusivity hypothesis), we introduce two variations on the original design which allows information contained within each memory to be explicitly assessed at recall.

In total, we employ three different conditions with each differing in terms of the stimuli presentation. The first type of stimulus presentation, which is a direct replication of one of Baguley et al.'s (2006) conditions, is a paired-single anchor presentation followed by dual-anchor test (PSA-DA). This condition allows for the encoding of two separate but related memories and tests for the combined performance of both memories. The learning phase consists of presenting a target object $(\mathrm{T})$ in the same location twice; once in relation to an anchor point (A) to the left of the target (A-T) (referred to as a single anchor-left [SA-L]) and once in relation to an anchor point to the right of the target (T-A) (referred to as a single anchor-right [SA-R]). Paired anchor points (i.e., those that share the same target object) are presented at different times and in a random manner with one caveat that they are not presented successively. In essence, each participant sees the same target in the same location but at two different times and in relation to two different anchor points. This manner of anchor presentation creates two representations for a single target object. The test condition involves simultaneously presenting the two learnt anchor points (A-T \& T-A) as cues to prompt memory of the target object's location (this is known as dual anchor cueing [DA].

The second condition consists of paired single anchor presentation followed by a paired single anchor test (PSA-PSA). In essence, it adopted the same learning phase as PSA-DA, in that each memory is encoded separately, however at test each memory was assessed separately (see Figure 1). This means the level of spatial information contained within each memory (as opposed to their combined performance) could be examined.

The third condition comprised dual anchor presentation followed by a paired single anchor test (DA-PSA) (see Figure 1). This condition shared the same test presentation as PSA-PSA but introduced a new encoding presentation. During encoding participants were presented with both anchor points along with the target simultaneously (as opposed to separately as in the other two conditions). Essentially the participant will encode an already integrated view of the two anchors and target. At test both anchors (i.e., cues) are presented separately. Again like PSA-PSA, the DA-PSA test phase allows for measurement of spatial information contained within each memory separately ${ }^{3}$.

For each condition there were nine targets to be remembered in nine unique locations (an example template of nine discrete locations with left and right anchors can be seen in

\footnotetext{
${ }^{3}$ It should be noted that a DA-DA condition has already been examined by Baguley et al. (2006) and found to demonstrate similar memory performance to that of the PSA-DA condition [see Figure 4 of Baguley et al. (2006)]
} 
THOMAS J. DUNN

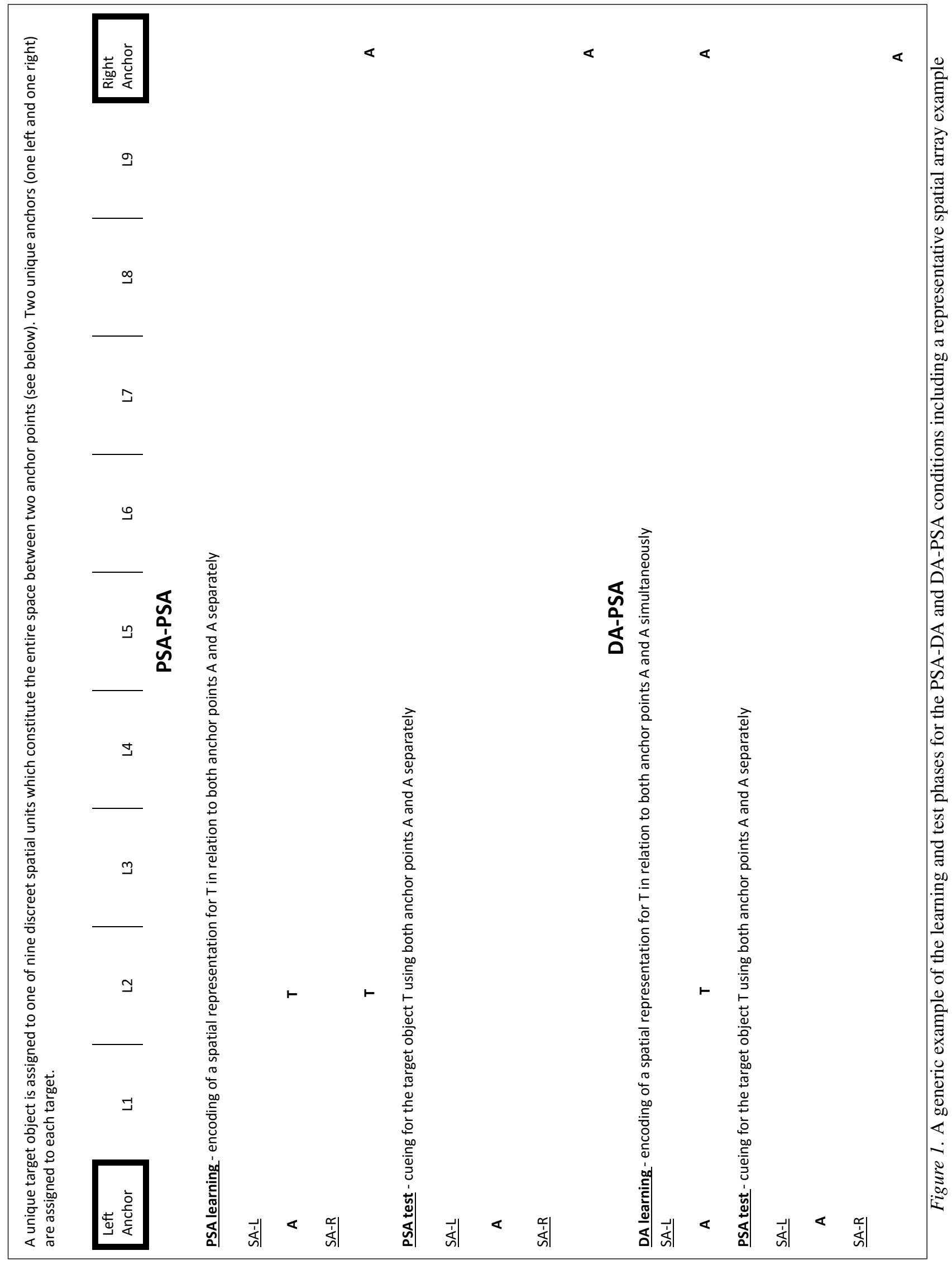


Figure 1). A single trial is created by presenting a target object with its corresponding anchor point(s) simultaneously. This meant because the target was presented twice during encoding for the PSA-PSA and PSA-DA conditions (once in relation to an anchor to the left and once in relation to a target on the right), the learning phase consisted of eighteen trials (nine left and nine right). However, because anchors were presented simultaneously for the DA-PSA condition the learning phase was made up of nine trials. ${ }^{4}$

\section{Measuring spatial memory and testing for exclusivity}

Accuracy of location memory was measured in terms of the number of quantifiable units (i.e., distance) a response is observed to be from its actual location (i.e., the number of unit deviations). The measurement of location memory is a chance-corrected score (Dscore) which adjusts for levels of chance error dependent on a target's location within the horizontal array (see Clark et al., 2013, for a detailed description of how Dscore is calculated). Dscore is better than an absolute measure because it incorporates error/near miss information. It follows that a lower Dscore (i.e., less error) equates to better location memory performance. A Dscore of 1 indicates performance is at chance and a Dscore of 0 indicates that memory is exact (i.e., that the participant has placed the target in the same discrete location that it was presented in). Dscores between 1 and 0 thus reflect increasing levels of accuracy in locating the target.

\section{Stimuli}

Words were employed as both anchor points and target objects in order to replicate previous research examining exclusivity (see Clark et al., 2013). The materials consisted of a pool of 270 words constituting 15 categories each containing 18 words. From this pool, categorically-defined groups of words were selected for use in the three conditions. Different categories were used for each condition, for example, PSAPSA $=$ musical instruments, DA-PSA = birds, PSA-DA = countries. Different categories were used to reduce any interference between conditions. Both order of conditions and category-condition pairings were counterbalanced. All target words were deemed to be semantically neutral to the anchor word(s) and were matched on average length, level of semantics, imagery, and pronunciation (Paivio et al., 1968). The 15 categories ranged from 'types of Animals' to 'US States'. The categorical words acted as anchor points and the neutral words were randomly allocated to these anchors to act as target objects. Neutral target words (i.e., not semantically related to the anchor words) were selected to possess high imagery $($ Mean $=5.9, S D=0.70$ out of a possible range 1-7) and high meaningfulness (Mean $=6.7, S D=0.80$ out of a possible range 1-10) values (Paivio et al., 1968).

\section{Participants and procedure}

Participants were recruited from Nottingham Trent University $(N=62)$. Each participant received research credits for their time. The experiment employed a within-subject design and was carried out on a computer using E-prime(c) software. Subjects were presented with a series of anchorword trials. Each trial consisted of a target word located a specified distance from an anchor word at a fixed location (either to the left and/or right hand side of the screen depending upon condition) and lasted for 4 seconds. The subjects were instructed to remember where the target word was located in relation to the anchor word. In between the learning and test phase a short distractor task (30 seconds) was employed (counting backwards in 3's) to prevent any short-term memory rehearsal. At test, participants clicked on the screen using a mouse where they remembered the corresponding target word to be located. The order in which participants carried out the conditions was counterbalanced. The order of stimuli presentation within each condition was randomised. To preclude participants from using any external anchor points/cues other than those manipulated in the study (e.g., the edge of the computer screen), the stimuli were presented on a black computer screen (with text appearing in white) and the lights were switched off in the room producing a near black backdrop.

\section{Results}

The results show performance in all conditions to be better than chance (i.e., Dscore $<1$ ) which indicates some information for object location. The mean Dscore for the PSADA condition was $0.74(S D=0.26)$. For the DA-PSA condition mean Dscore was $0.83(S D=0.23)$. The PSA-PSA mean Dscore was $0.80(S D=0.23)$. To test the differences in mean Dscore performance across all three anchor conditions a linear model was estimated with Anchor type predicting Dscore. The results show that the overall model did not quite reach significance (Mean difference [Dscore] $=-0.029, S E$ $=0.017, t=-1.76, \mathrm{p}=0.078)$, which suggests there was no difference in terms of overall memory performance across the three conditions.

\section{Examining memory performance for each separate spa- tial memory}

This section reports further analysis of both PSA-cueing conditions from the PSA-PSA and DA-PSA conditions. Analysis of each related memory from the PSA-PSA and DA-PSA conditions showed that location information regarding a common target was better than chance for both

\footnotetext{
${ }^{4}$ It should be noted that previous findings (see Experiment 3 Baguley et al., 2006) show that the disparity in the number of encoding trials between conditions cannot sufficiently account for memory performance.
} 
memories. This indicates that location information is encoded and available at recall when prompted with each corresponding cue. This suggests that exclusive encoding and/or retrieval of two related spatial memories may not fully account for the equal levels of performance from having one or two spatial memories. In other words, if only one of two memories was being encoded or retrieved (i.e., processed exclusively) then this should be reflected in the amount of spatial information contained within each memory. In particular, some kind of disparity in terms of memory performance between each memory should be apparent (e.g., one memory being dominant and significantly more accurate than the other). Under the current analysis, the similar levels of spatial information observed in both retrieved memories suggest both are encoded and are later available to be retrieved nonexclusively.

\section{Modelling memory performance for all cueing conditions}

The first part of this section focuses on modelling memory performance for both conditions which allowed, for the first time, explicit examination of spatial information contained within each memory separately (PSA-PSA and DA-PSA). The next part examines patterns of response when both cues are presented concurrently, as has been employed in previous research (Baguley et al., 2006; Clark et al., 2013).

Dscore was modelled for left and right anchors for both PSA-PSA and DA-PSA. Linear models were specified to capture the relationship between memory accuracy (i.e., Dscore) and location of the target relative to an anchor point (i.e., location). This was carried out for both related anchor points (left and right) for both PSA-cueing conditions (PSAPSA and DA-PSA). Parameter estimates for the PSA-PSA and DA-PSA conditions can be seen in Table 1.

The pattern of memory performance is quite similar across locations 1-9 for both conditions (see Figure 2). That is, they both demonstrate a relationship between Dscore and location, in that, memory for an object's location increases in error (reduces in accuracy) the further away the target is from the anchor point (see Nelson \& Chaiklin, 1980; Huttenlocher, Hedges, \& Duncan, 1991, for an explanation of distance and accuracy trade-off). Plots showing the pattern of responses for left and right anchor points across locations can be seen in Figure 2.

It is clear from examination of errors (i.e., Dscores) that the accuracy of spatial information varies in an almost opposing pattern across left and right anchor points. This relationship is apparent for both conditions where such examination is possible (i.e., PSA-cueing conditions). This is interesting because it suggests that an individual has encoded the target object in relation to both anchor points separately and that each memory will vary inversely in terms of location accuracy for a common target. To take the most extreme example, if a target were presented in location one (see Figure 2 for an example) this would result in one relatively precise memory, associated with the left anchor point, and another relatively imprecise memory, associated with the right anchor point. This highlights that at least up until the point of simultaneous cueing, a participant has two memories available (albeit varying in accuracy) upon which to draw. This suggests that simultaneous cueing may modify memories via the application of a strategy (e.g., averaging or exclusive retrieval). Examining the pattern of response error for such a circumstance might help to inform us of just how two such memories are utilised. In light of this, the next section focuses on examination of the responses from the PSA-DA condition, where memories are cued simultaneously.

\section{Analysing memory performance for the PSA-DA condi- tion}

A scatter plot representing memory performance across location (see Figure 4) for two anchor points presented simultaneously at test (i.e., PSA-DA) shows a different pattern of errors compared to cueing each memory separately (see Figure 2). The relationship between memory performance and target location was assessed by modelling Dscore as a function of target location. Two models were specified, an intercept only model which would not allow the slope to vary (i.e., representing no relationship between Dscore and location) and a linear model with Dscore being predicted by location. The results showed the intercept only model was a marginally better fit of the data and is represented in Figure 4. When comparing the responses for either of the PSA-cueing conditions with those of the PSA-DA condition is that performance for PSA-DA appears to equate to an averaging of SAL and SAR information from the PSA-cueing conditions. This is in line with an averaging or non-beneficial combination account of memory performance. This idea can be represented graphically by averaging the SAL and SAR responses for every location in each PSA-cueing condition and then modelling those averages. Figure 4 shows how close an averaging model would approximate levels of memory performance if subjects were cued simultaneously. To formally test this, a linear model was estimated using an interaction term (Cueing type [DA, average of PSA] x Location) to establish whether dual cueing and the average of PSA cueing differed in Dscore as a function of location. Results show no significant interaction between cue-type (DA vs. average of PSA) and location ( $\beta=0.02, S E=0.02, t=1.03, \mathrm{p}=$ 0.32 ). This suggests that if information were averaged from two related memories (SA-L \& SA-R), it would approximate the performance observed when memories are cued simultaneously.

In order to explore this further, a number of hypotheses can be tested. The first is that memories are averaged based on precision, where the more precise memory is given more weight. The second is that memories are averaged based 
Figure 2. Average Dscore by location for PSA-PSA and DA-PSA conditions arranged by anchor (left vs. right)

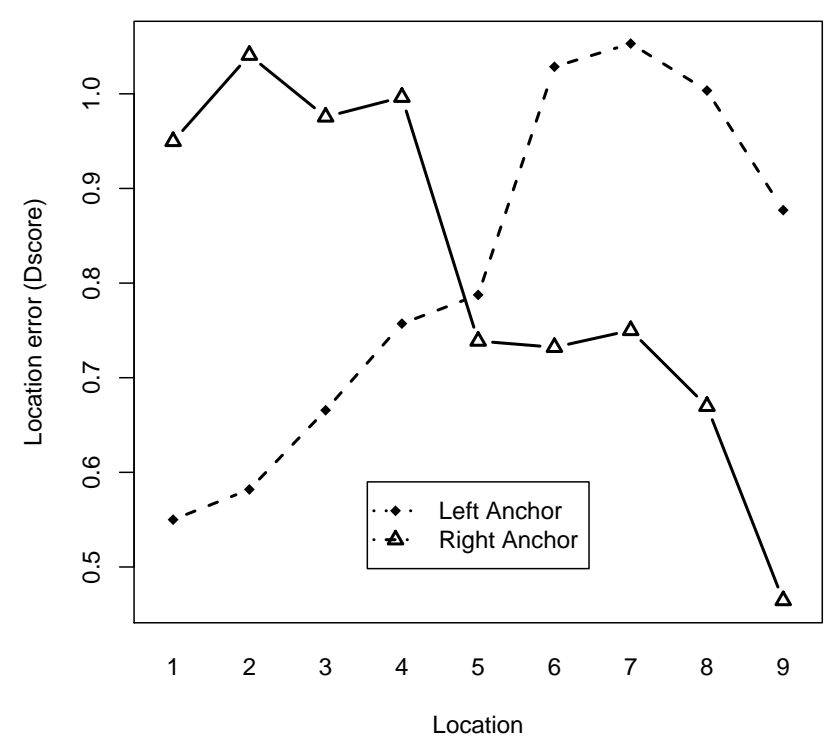

(a) PSA-PSA

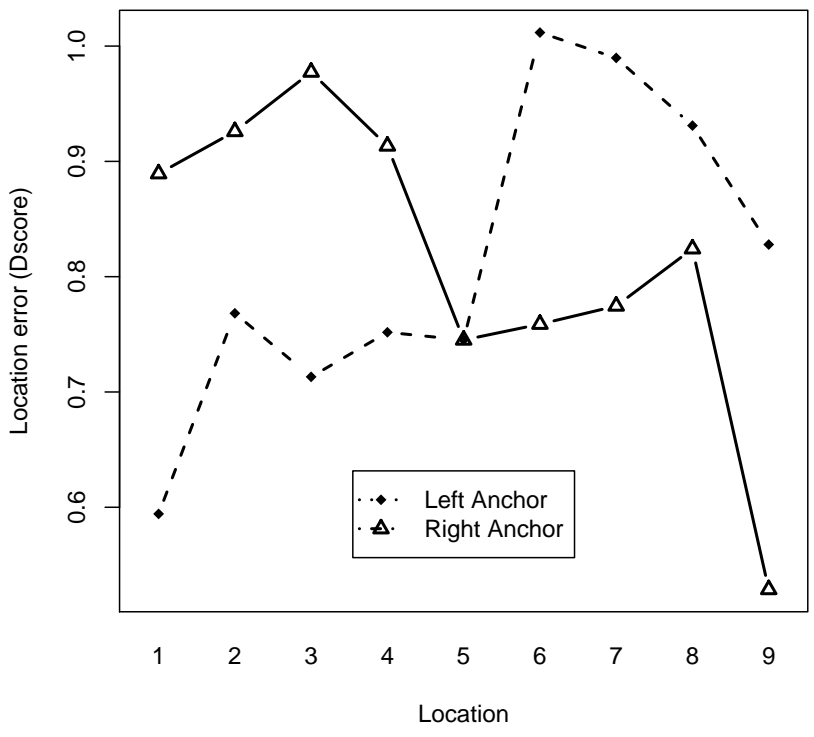

(b) DA-PSA

Figure 4. Average Dscore by location for the PSA-DA condition. The plot also includes linear models representing PSA-DA performance and an averaging model comprised by averaging left and right anchors from PSA-PSA \& DA-PSA conditions

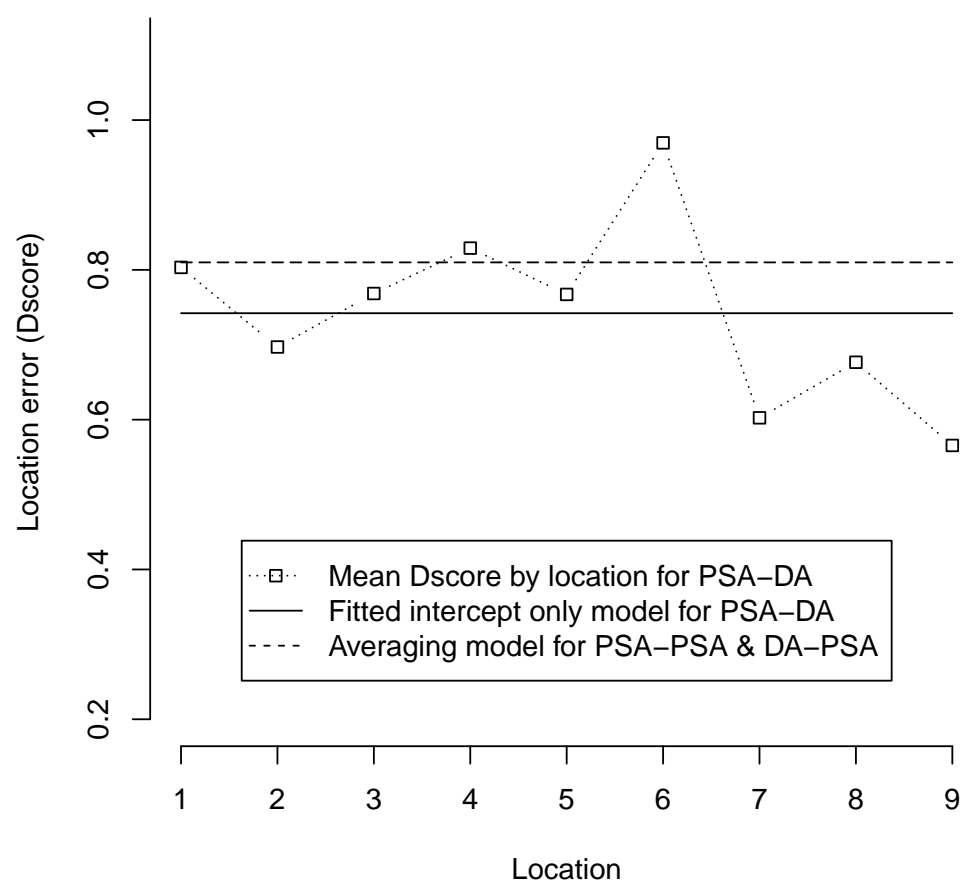


THOMAS J. DUNN

Table 1

Summary of parameter estimates - Dscore predicted by Target location for SAL and SAR

\begin{tabular}{rrrrrr}
\hline \hline & Estimate & $S E$ & $t$-value & $p$-value & $R^{2}$ (adj.) \\
\hline PSA-PSA & & & & & \\
(Intercept) & 0.51 & 0.04 & 11.88 & & \\
SAL & 0.06 & 0.01 & 7.91 & $<0.01$ & $0.06(0.05)$ \\
(Intercept) & 1.02 & 0.04 & 26.99 & & \\
SAR & -0.06 & 0.01 & -8.45 & $<0.01$ & $0.06(0.06)$ \\
\hline DA-PSA & & & & & \\
(Intercept) & 0.63 & 0.06 & 10.47 & & \\
SAL & 0.04 & 0.01 & 3.49 & $<0.01$ & $0.02(0.02)$ \\
(Intercept) & 1.01 & 0.06 & 17.23 & & \\
SAR & -0.04 & 0.01 & -3.70 & $<0.01$ & $0.02(0.02)$ \\
\hline \hline
\end{tabular}

Figure 5. Linear model representing directional bias across targets with SE. Note: Positive y-axis values indicate that memory is biased to the left of the target, negative values indicate it is biased to the right

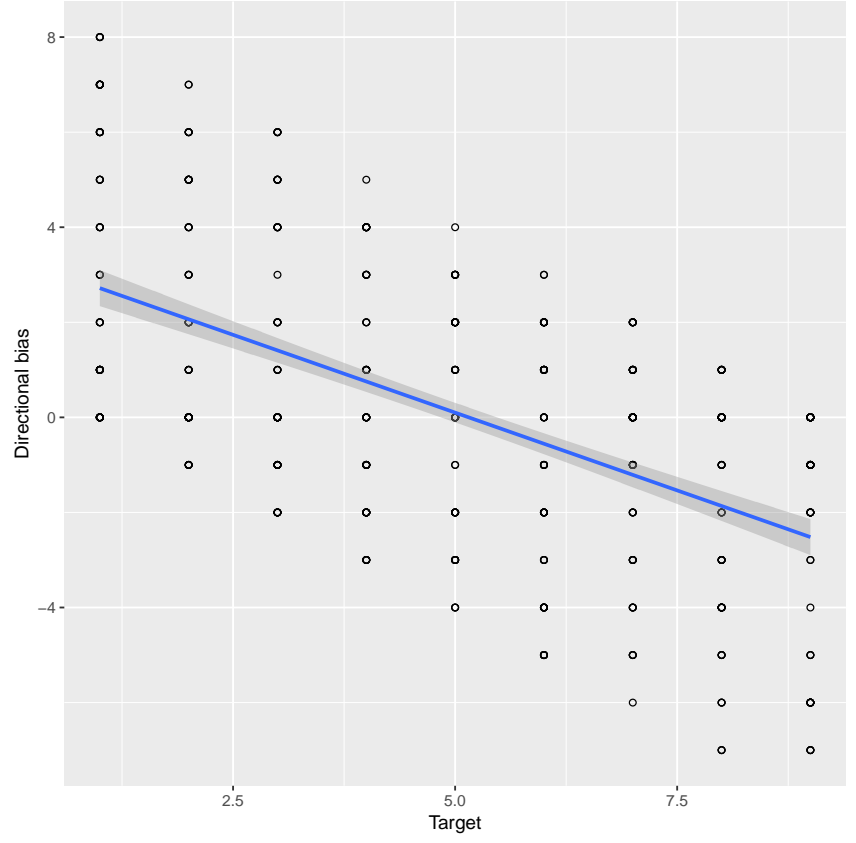

on spatial quantity (i.e., euclidean distance from the anchor point to target), with the largest quantity being given more weight. These two possibilities would lead to opposing response patterns and can be tested by modelling the relationship of signed error (the direction of a response in relation to the target) across target locations. If memories are averaged by weighting precision, then a response bias should be observed with the direction tending towards the memory with greatest precision (i.e., the anchor point closest to the target). For example, a target placed in location two would result in a more precise memory for the left anchor than the right, because it is simply closer to the left anchor point. In this case, the response at cueing should be biased toward the left anchor (i.e., responses will systematically be left of the target). However, if memories are averaged by weighting spa- tial quantity (i.e., euclidean distance from the anchor point to target) then the opposite should occur and a directional bias towards the largest spatial quantity be observed (i.e., right of the target).

Results from a linear regression confirm a pattern of response bias in line with a spatial quantity averaging model $\left(\beta=-0.65, S E=0.04, t=-16.17, p<0.01, R^{2}=0.33\right)$. Specifically, it shows that for target locations one, two, three and four the directional bias is positive, meaning the target is systematically estimated to be right of its actual location (see Figure 5). In contrast, for locations six, seven, eight and nine the directional bias is negative, meaning the target is systematically estimated to be right of its actual location. In line with this, location five (i.e., the central location equidistant from either anchor point) shows no directional 
bias. This highlights a number of relevant findings. First, it suggests that information from both memories is utilised during estimation of a common target's location, in contrast to the exclusivity hypothesis. Second, it suggests that information is not combined by way of weighting the memory with most precision (i.e., smallest error) but rather in terms of the largest spatial quantity (distance from anchor point).

\section{Discussion}

\section{An exclusivity account}

Previous work suggests that when given the opportunity to encode or retrieve two separate spatial memories, only one is relied upon. The exclusive reliance on a single spatial representation is thought to account for similar spatial judgement performance when holding one compared with two spatial memories. Through the adaptation of a previous experimental design, the current research has the exclusivity hypothesis more rigorously. One adaptation in particular held the encoding phase constant with that of previous research (e.g., PSA-DA) but varied the test phase to allow for examination of each encoded memory (i.e., PSA-PSA). The results from this manipulation showed location information to be better than chance and to be equally available across both memories. This provides strong evidence that when given the opportunity to encode the location of a target object in relation to two different anchor points, the target is encoded in relation to both and results in two representations being available. This supports previous findings, using a comparative judgement task (Lansdale et al., 2013), that exclusivity does not likely occur at encoding.

The fact that information is available from both memories for the DA-PSA condition suggests that when presented with a target object and two anchor points together at learning, information from each memory is available at test to help relocate a target's location. This suggests that during encoding either the target object is explicitly encoded twice once in relation to each anchor (much in the same way as PSAlearning forces participants to do in the PSA-DA or PSAPSA conditions), or the target object as well as both anchor points are encoded as one holistic representation. Due to the lack of any performance difference between PSA-PSA and DA-PSA conditions it suggests the same mechanisms underlie encoding in both conditions (based on the assumption that transformational effort is required to extract separate memories from an existing combined representation). However, further research is needed to substantiate which possibility is more likely.

Whether or not exclusive retrieval can account for similar performance between holding one or two memories (i.e., PSA-DA) is perhaps more difficult to discern based on the current findings. It could be argued that exclusive processing is occurring at retrieval with the simultaneous presenta- tion of cues, as is present in the PSA-DA condition (e.g., concurrent cueing might create a bottleneck or blocking between cues). This is difficult to establish because cues are presented simultaneously and force a single response which precludes the possibility to measure information contained within each memory separately. However, the fact that information is present within both memories (i.e., as was possible to ascertain in the PSA-PSA \& DA-PSA conditions), suggests that the retrieval of information would occur similarly in the PSA-DA condition and hence be available from both cues separately, even when a target is being cued simultaneously. Additionally, bias analysis (discussed in more detail below) suggests that both anchor points are acting to influence the estimation of a target object's location. Overall, the findings are generally inconsistent with the idea of exclusive processing and suggest the need to explore an alternative explanation.

\section{An averaging account}

In addition to demonstrating findings which seem to be incompatible with the idea of exclusive processing, the current study also explored an averaging model to account for previous findings. Interestingly, results show a striking difference in spatial estimation errors between single anchor cueing conditions (i.e., PSA-L \& PSA-R) and a dual cueing condition (i.e., PSA-DA). Upon modelling these patterns, it was shown that separate cueing of a target's location produces a monotonically increasing relationship between location (i.e., distance from anchor point) and accuracy, whereas simultaneous cueing produces no relationship between location and accuracy. That is, the distance of the target from the anchor does not seem to impact the accuracy of a response when two related anchor points are available.

One conclusion that could be drawn from this is that information from both memories is being relied upon at recall when two memories are cued simultaneously. A result of this reliance disrupts the relationship between accuracy and target location, as is observed when only one memory is cued. Owing to the fact that there is no observable difference from holding two spatial memories, this suggests that combining of information may occur in way that does not allow for either beneficial integration or produce a significant degradation of performance, compared to that of a single memory. This means that greater location accuracy contained within one memory (i.e., the memory where the target is closest to the anchor) is being cancelled out by combining it with nonuseful information contained within an opposing memory. A result of this process is that performance approximates the average of two spatial memories (i.e., or the same as a single memory).

In line with the findings that show a systematic bias in recall, one possibility is that some form of averaging is taking place that does not rely on weighting memories in terms of 
precision but rather in terms of spatial quantity (i.e., distance from the anchor point). In this regard, location estimation is biased towards the larger spatial quantity. This biasing appears to off-set any potential performance gains expected if only the most accurate memory were sampled (i.e., the memory closest to the anchor point). Such a bias might be deemed beneficial under circumstances of uncertainty as it would be advantageous to abate the possibility of making an extreme error. In the current context, because the memory with the largest spatial quantity offers the greatest potential for error, it makes sense for a response to be biased towards the centre of the largest spatial quantity. However, in doing so this has a knock-on effect of biasing a response away from a potentially more accurate memory, resulting in attenuated levels of performance akin to relying on a single memory.

\section{To integrate or to average?}

Two mechanisms could help explain why averaging might happen. The first concerns the prevention of integration, the second the optimisation of averaging.

Under a Bayesian-type approach, when two sources of information stem from different signals it becomes inefficient and unreasonable to try to integrate them (Cheng et al., 2007). In the same way that unique egocentric perspectives represent different signals (Cheng et al., 2007), the two memory representations in the current task could also be said to stem from different signals. Discrepant spatial information across representations in the form of spatial quantity (i.e., distance from anchor point) and directional information (leftto-right vs. right-to-left) may make deciphering signal certainty untenable. In other words, the two memory representations may contain incompatible information that discourage the formation of a qualitatively better single representation. A disparity in spatial quantity and directional information may require additional operations (e.g., rotation or comparison) necessary for beneficial integration, thus making it an inefficient method of representing spatial information. Testing these ideas further would require diminishing signal uncertainty by way of reducing the incongruence of spatial quantity and directional information.

A second underlying process encourages the averaging of current and prior information. If uncertainty is present regarding a current source, past experiences are averaged and relied upon (Cheng et al., 2007). This type of averaging occurs in other domains of multiple-source processing where uncertainty is present. For example, the McGurk effect describes a phenomenon where visual and auditory information are combined to form an average of both sources, with no obvious benefit. Lansdale et al.'s (2013) idea that precision and availability of information impact the way memories interact can be reconciled somewhat with the idea of averaging. In the current task, uncertainty might stem from source uncertainty or cue competition at recall such as overshadowing
(Pavlov, 1927), both of which may lead to a degradation of precision. Notwithstanding, Lansdale et al. (2013) suppose that this impacts a balance in memory function between exclusivity and additivity. In contrast, an averaging approach would suggest that when precision is high (i.e., source uncertainty low), integration (additivity) would be the best strategy. However, when precision is low (i.e., uncertainty high) averaging of sources is preferred. Thus, an averaging approach supposes the balance in memory function occurs between averaging (rather than exclusivity) and additivity. It makes sense that when two sources are equally available but subject to uncertainty, that combining them through averaging can reduce the probability of making extreme errors. This may well be optimal because memory systems have evolved to provide the most consistently useful map over time (i.e. on one exposure where there is uncertainty in precision the best outcome might come by building from multiple cues).

The possibility of averaging spatial memories is particularly interesting because much of the research examining integration (whether spatial or of another type), generally assumes that the combining of information will lead to performance gains (Jones, 1987; Bryant \& Subbiah, 1994; Lindberg \& Garling, 1987). This is typically based on the premise that integration results in the summing of useful information or that a qualitatively better representation is formed. Although research does observe gains employing similar paradigms (Rubin \& Wallace, 1989), it might not necessarily be the case that benefits of integration hold true across different situations. After all, previous research that differs in terms of integrative advantages also vary by way of stimuli, types of memory, and scale of space involved (e.g., associative memory Rubin \& Wallace, 1989). Thus, information specific to a task may determine the possibility of combining information in an advantageous manner.

\section{Future research: is integration possible?}

An averaging approach highlights conditions that may be more conducive to integration. These include reducing discrepancies in spatial quantity or opposing directional information across memories and thus increasing the overlap (i.e., common signal) between two sources. Future research should focus on enhancing such conditions for tasks similar to that of Baguley et al. (2006), perhaps through multiple exposure to stimuli over time or by using cues that are equidistant from the target (i.e., either both on the left or right of the target).

The current research highlights the importance to differentiate between beneficial combining of information (e.g., integration in the traditional sense) and non-beneficial combining of information (e.g., averaging of precision and error). Admittedly testing such possibilities may prove difficult, nevertheless as a theoretical standpoint it should not be ignored in future research. This could be considered in other 
domains of memory where exclusivity has been observed (e.g., semantic and autobiographical memory, see Maylor, Chater, \& Jones, 2001).

\section{Conclusion}

Using a similar task to that where exclusivity was first observed, this research suggests exclusive processing may not be the most appropriate explanation of previous findings. In light of this, the current article sets out an alternative explanation for why two sources of spatial information perform only as well as a single source. At least for small-scale figural space, multiple spatial memories might interact in a way that combines useful (precision) with non-useful information (error in the form of bias towards the largest spatial quantity) to reduce the possibility of making an extreme error. The underlying reason for this could stem from the high levels of incompatible information contained within each representation making beneficial integration burdensome. In sum, an alternative explanation to exclusivity, in the form of an averaging model, is offered which accounts for the presence of information in each encoded memory as well as why two memories are sometimes only as good as one.

\section{References}

Alvarez, G. A., \& Cavanagh, P. (2004). The capacity of visual short-term memory is set both by visual information load and by number of objects. Psychological Science, 15(2), 106-111.

Baguley, T., Lansdale, M. W., Lines, L. K., \& Parkin, J. K. (2006). Two spatial memories are not better than one: Evidence of exclusivity in memory for object location. Cognitive Psychology, 52(3), 243-289.

Bryant, D., \& Subbiah, I. (1994). Subjective landmarks in perception and memory for spatial location. Canadian Journal of Experimental Psychology-Revue Canadienne De Psychologie Experimentale, 48(1), 119-139.

Cheng, K., Shettleworth, S. J., Huttenlocher, J., \& Rieser, J. J. (2007). Bayesian integration of spatial information. Psychological bulletin, 133(4), 625-637.

Clark, D. P. A., Dunn, A. K., \& Baguley, T. (2013). Testing the exclusivity effect in location memory. Memory, 21, $512-523$

Greenauer, N., Mello, C., Kelly, J. W., \& Avraamides, M. N. (2013, SEP 2013). Integrating spatial information across experiences. Psychological Research-
Psychologische Forschung, 77(5), 540-554. (PT: J; TC: 1; UT: WOS:000323254600003)

Hollingworth, A., \& Henderson, J. M. (2002). Accurate visual memory for previously attended objects in natural scenes. Journal of Experimental Psychology: Human Perception and Performance, 28(1), 113-136.

Hubbard, T. L., \& Ruppel, S. E. (2000). Spatial memory averaging, the landmark attraction effect, and representational gravity. Psychological ResearchPsychologische Forschung, 64(1), 41-55.

Huttenlocher, J., Hedges, L. V., \& Duncan, S. (1991). Categories and particulars: Prototype effects in estimating spatial location. Psychological Review, 98(3), 352376.

Jones, G. V. (1987). Independence and exclusivity among psychological processes: Implications for the structure of recall. Psychological Review, 94(2), 229-235.

Lansdale, M., Humphries, J., \& Flynn, V. (2013). Cognitive operations on space and their impact on the precision of location memory. Journal of Experimental Psychology: Learning, Memory, and Cognition, 39(5), 1501 1519.

Lindberg, E., \& Garling, T. (1987). Memory for spatial location in two-dimensional arrays. Acta Psychologica, 64(2), 151-166.

Maylor, E. A., Chater, N., \& Jones, G. V. (2001). Searching for two things at once: Evidence of exclusivity in semantic and autobiographical memory retrieval. Memory $\mathcal{E}$ Cognition, 29(8), 1185-1195.

Molet, M., Bugallo, M., \& Gambet, B. (2011). Spatial integration using a $3 \mathrm{~d}$ virtual environment with humans. Behavioural processes, 88(3), 198-201.

Molet, M., Gambet, B., Bugallo, M., \& Miller, R. R. (2012). Spatial integration under contextual control in a virtual environment. Learning and motivation, 43(1-2), 1-7.

Nelson, T. O., \& Chaiklin, S. (1980). Immediate memory for spatial location. Journal of Experimental Psychology: Human Learning and Memory, 6(5), 529-545.

Pavlov, I. P. (1927). Conditioned reflexes. Oxford, England: Oxford University Press.

Rubin, D. C., \& Wallace, W. T. (1989). Rhyme and reason: Analyses of dual retrieval cues. Journal of Experimental Psychology: Learning, Memory, and Cognition, 15(4), 698-709. 